Mathematical Modelling and Analysis

Volume 21 Number 2, March 2016, 159-173

http://dx.doi.org/10.3846/13926292.2016.1142481

(C) Vilnius Gediminas Technical University, 2016
Publisher: Taylor\&Francis and VGTU

http://www.tandfonline.com/TMMA

ISSN: $1392-6292$

eISSN: 1648-3510

\title{
Strong Stability of a Nonlinear Multi-Stage Dynamic System in Batch Culture of Glycerol Bioconversion to 1,3-Propanediol
}

\section{Jinxing Zhang ${ }^{a, b}$, Jinlong Yuan ${ }^{a, b, c}$, Enmin Feng ${ }^{a}$, Hongchao $\mathrm{Yin}^{b}$ and Zhilong $\mathrm{Xiu}^{c}$}

\author{
${ }^{a}$ School of Mathematical Sciences, Dalian University of Technology \\ ${ }^{b}$ School of Energy and Power Engineering, Dalian University of Technology \\ ${ }^{c}$ School of Life Science and Biotechnology, Dalian University of Technology \\ Dalian, 116024 Liaoning, China \\ E-mail(corresp.): yuanjinlong06130163.com \\ E-mail: jinxingzhangdlut@163.com \\ E-mail: emfeng@dlut.edu.cn \\ E-mail: hcyin@dlut.edu.cn \\ E-mail: zhlxiu@dlut.edu.cn
}

Received August 3, 2015; revised January 11, 2016; published online March 15, 2016

\begin{abstract}
In this paper, we consider a nonlinear multi-stage dynamic system to characterize batch culture. We construct corresponding linear variational system for the solution to the multi-stage system, also prove the boundedness of fundamental matrix solutions for the linear variational system. On this basis, we prove strong stability with respect to perturbance of initial state vector for the multi-stage system through the application of such boundedness. From extensive simulation study, it is observed that the strong stability is highly satisfactory.

Keywords: nonlinear multi-stage system, linear variational system, fundamental matrix solution, strong stability.
\end{abstract}

AMS Subject Classification: 37C75; 93C10.

\section{Introduction}

1,3-Propanediol (1,3-PD) has a wide range of potential application on a large commercial scale, especially as one of the bulk chemicals used as a monomer for polyester, polyethers and polyurethanes [26]. Production methods for 1,3PD can be one of two categories: chemical synthesis and microbial conversion of glycerol by Klebsiella pneumonia (K. pneumonia) [25]. This paper focuses on the latter category, which has recently come into investigators attention throughout the world because of no harm to environment, high region specificity and cheaply renewable feedstock [6]. In microbial fermentation process 
of glycerol bioconversion to 1,3-PD, a number of models were proposed to describe the process $[24,29]$. The fermentation process can be one of three types: either batch culture (all substrate is present at the beginning of the reaction and nothing is added or removed from the fermentor during the reaction); fedbatch culture (fresh medium is added during the reaction to prevent nutrient depletion, but nothing is removed); and continuous culture (fresh medium is added during the reaction while old medium is removed) [17]. A comparison of batch culture with continuous and fed-batch cultures has recently been come into investigators notice throughout the world as it can obtain the highest production concentration and molar yield 1,3-PD to glycerol [1]. Not only the nonlinear dynamic behaviour of microorganisms has been the objective of a number of theoretical works, but also much modelling research on the process of batch culture has been reported recently in $[2,16,23,28]$. However, for all the papers mentioned above, the strong stability is not taken into consideration in batch culture.

There is a long history in stability study of dynamical system. Many theoretical and applied researchers are investigated by mathematicians, physicists, astronomers and engineering professionals $[9,11,22]$. The rule of minimum total energy, local point of which was stable and balanceable, was proposed by Torricelli in 17th century. This rule is expanded by Laplace and Lagrange, both of which believe that the state of the corresponding zero kinetic energy and minimum potential energy was stable and balanceable if system was conservative in 18th century. The general theory and method of motive stability was studied by Lyapunov in 19th century. The invariance theorem was considered by LaSalle in 20th century. Generally, ranges of parameters are restricted in the neighborhood of initial parameter values during the identification process [18]. Because we can not ensure the system is stable under the given ranges of parameters, stability of the system becomes a fundamental issue in system analysis and design [27]. Stability theory was started by Morley [19], who introduced several of the fundamental concepts, such as totally transcendental theories and the Morley rank. Stable and superstable theories were first introduced by Shelah [20], who was responsible for much of the development of stability theory. The definitive reference for stability theory was proposed by Shelah [21], though it is notoriously hard even for experts to read. Zhao [30] discussed stability of impulsive system by perturbing Lyapunov functions. Gao $[7,8]$ studied the approximate stabilisation and reachability of uncertain hybrid systems. Li [15] researched the stability analysis of dynamic collaboration model with control signals on two lanes. Fridman [5] investigated stability of linear descriptor systems with delay through the application of a Lyapunov-based approach by introducing its linearization. Chio $[3,4]$ researched the stability of linear and nonlinear dynamic system using the notion of fundamental matrix solution and integral Gronwall's inequality. The stability of equilibrium solutions for nonlinear dynamic system was discussed by introducing its linearization in continuous culture $[13,14,27]$. Nevertheless, there is no equilibrium point for nonlinear dynamic system in batch culture. Therefore, the general approaches fail to obtain the stability of the system in which the conditions to the existence of equilibrium point are untenable. 
In this paper, to describe the process of batch culture of glycerol biconversion to 1,3-PD induced by $K$. pneumoniae, we consider a nonlinear multi-stage dynamic system in which there is no equilibrium point. Some properties of the nonlinear system are discussed. Our goal is to prove the strong stability with respect to perturbance of initial state vector for the solution of the multi-stage system. For this, we establish corresponding linear variational system for the solution to the multi-stage system and show the boundedness to fundamental matrix solution of the linear variational system. After this works, we state and prove the above strong stability through the application of such boundedness. The numerical simulations are provided to confirm the theoretical results.

The rest of this paper is organized as follows. In Section 2, a nonlinear multi-stage dynamic system is considered and some important properties of the multi-stage system are discussed. In Section 3, we construct corresponding linear variational system for the multi-stage system and prove the boundedness of its fundamental matrix solution. In Section 4, we demonstrate the strong stability with respect to perturbance of initial state for the solution of the multi-stage system. In Section 5, numerical simulation of a batch experiment is carried out. In Section 6, we draw the conclusions and trace the direction for future works.

\section{Nonlinear multi-stage dynamic system and its properties}

Some researchers considered the fermentation process as only growth period, which neglected the distinctive impact of cells during different stages. In the lag stage, the cells will take time to adjust and the exponential stage is characterized by cell doubling. During the stationary stage growth will slow down and eventually stop as nutrients become depleted or inhibitory metabolites build up in this stage [28].

In the light of the actual experiment, the following assumptions will be in force throughout the rest of this paper, whether explicitly mentioned or not.

- A1. No medium is pumped inside and outside the bioreactor in the process of batch culture; and

- A2. The concentrations of reactants are uniform in reactor, while nonuniform space distribution is ignored.

Next we describe the main nomenclature: $\mathbb{R}_{+}$denotes the set of positive real numbers, $\mathbb{N}_{+}$denotes the set of positive integers, $I_{n}$ denotes the set $\{1,2, \ldots, n\}, n \in \mathbb{N}_{+}, A^{\mathrm{T}}$ denotes the transposition of the vector or matrix $A, t_{f}$ denotes the terminal time of the fermentation process, $t_{f_{1}}$ and $t_{f_{2}}$ are the given optimal parameters, $x^{i}(t):=\left[x_{1}^{i}(t), \ldots, x_{5}^{i}(t)\right]^{\mathrm{T}} \in \mathbb{R}_{+}^{5}$ is the state variable vector (whose components are the state variables) of the $i_{t h}$ stage at $t \in\left[t_{f_{i-1}}, t_{f_{i}}\right], i \in I_{3}, x^{1}\left(t_{0}\right)=x^{0}\left(t_{f_{0}}\right)=x_{0} \in \mathbb{R}_{+}^{5}$ is initial state vector of the first stage for the system $(2.1), x^{i-1}\left(t_{f_{i-1}}\right), i=2,3$ is both initial state vector of the $i_{t h}=2,3$ stage and end state of the $(i-1)_{t h}$ stage for the system $(2.1)$, 
The known parameters, the values of which are derived from [25], are defined as follows in batch culture.

- $K_{s}$ denotes the Monod saturation constant for substrate in $m m o l L^{-1}$.

- $Y_{s}$ and $Y_{j}, j=3,4,5$, denote the maximum biomass growth yield in gmmol $^{-1}$ and product (extracellular 1,3-PD, acetate, ethanol) yield in $m m o l g^{-1}$, respectively.

- $\mu_{m}$ denotes the maximum specific growth rate in $h^{-1}$.

- $m_{s}$ and $m_{j}, j=3,4,5$, denote maintenance term of substrate consumption and product (extracellular 1,3-PD, acetate, ethanol) formation under substrate-limited conditions in $\mathrm{mmolg}^{-1} h^{-1}$, respectively.

According to three different stages of batch culture $D_{0}:=\left[t_{0}, t_{f}\right] \subset \mathbb{R}_{+}$of glycerol to $1,3-\mathrm{PD}$, namely, the development stage (denoted by $D_{1}:=\left[t_{0}=\right.$ $\left.t_{f_{0}}, t_{f_{1}}\right] \subset \mathbb{R}_{+}$, or the first stage), the second or growth stage (denoted by $D_{2}:=$ $\left[t_{f_{1}}, t_{f_{2}}\right] \subset \mathbb{R}_{+}$) and the stabilization stage (denoted by $D_{3}:=\left[t_{f_{2}}, t_{f_{3}}=t_{f}\right] \subset \mathbb{R}_{+}$, or the third stage), we consider the nonlinear multi-stage system of batch culture (see [25]):

$$
\begin{cases}\dot{x}^{i}(t)=h^{i}\left(x^{i}(t)\right), & t \in D_{i}:=\left[t_{f_{i-1}}, t_{f_{i}}\right], i \in I_{3}, \\ x^{i}\left(t_{f_{i-1}}\right)= \begin{cases}x^{0}\left(t_{0}\right)=x_{0}, & i=1, \\ x^{i-1}\left(t_{f_{i-1}}\right), & i=2,3 .\end{cases} \end{cases}
$$

From [24], we have

$$
x_{*}=[0.01,150,0,0,0]^{\mathrm{T}}, x^{*}=[15,2039,939.5,1026,360.9]^{\mathrm{T}} .
$$

So the admissible range $W_{\alpha}$ of state vector $x^{i}(t)$ is

$$
W_{\alpha}=\left[x_{*}, x^{*}\right]:=\prod_{j=1}^{5}\left[x_{j *}, x_{j}^{*}\right] \subset \mathbb{R}_{+}^{5} .
$$

Based on the results in [25], the right term of the system (2.1) can be written as follows

$$
\begin{aligned}
h^{i}\left(x^{i}(t)\right):= & {\left[\mu(t) x_{1}^{i}(t),-q_{2}(t) x_{1}^{i}(t), q_{3}(t) x_{1}^{i}(t),\right.} \\
& \left.q_{4}(t) x_{1}^{i}(t), q_{5}(t) x_{1}^{i}(t)\right]^{\mathrm{T}} \in \mathbb{R}^{5}, \quad i \in I_{3},
\end{aligned}
$$

where

$$
\left\{\begin{aligned}
\mu(t) & =\mu_{m} \frac{x_{2}(t)}{x_{2}(t)+K_{s}} \prod_{j=2}^{5}\left(1-x_{j}(t) / x_{j}^{*}\right) \\
q_{2}(t) & =m_{s}+\mu(t) / Y_{s} \\
q_{j}(t) & =m_{j}+\mu(t) Y_{j}, j=3,4,5 .
\end{aligned}\right.
$$

From (2.3) to (2.4), we can directly prove 
Property 1. $h^{i}\left(x^{i}(t)\right)$, defined by (2.3), is measurable on $t \in D_{i}, i \in I_{3}$. Furthermore, $h^{i}\left(x^{i}\right)$ satisfies the linear growth condition, namely, there exists a constant $L>0$, such that $\left\|h^{i}\left(x^{i}(t)\right)\right\| \leq L\left(\left\|x^{i}(t)\right\|+1\right), \forall x^{i} \in W_{a}, i \in I_{3}$. In addition, $h^{i}\left(x^{i}(t)\right)$ is Lipschitz continuous in $x^{i}(t) \in W_{\alpha}, i \in I_{3}$.

According to the experiment process, we define the admissible set of initial state vector $x_{0} \in \mathbb{R}^{5}$, denoted by $W_{0} \subset \mathbb{R}_{+}^{5}$, namely,

$$
W_{0}:=[0.01,0.25] \times[150,520] \times\{0\} \times\{0\} \times\{0\} \subset \mathbb{R}_{+}^{5} .
$$

By Property 1 and the solution existence theorem of the ordinary differential equations, it is easy to prove that

Property 2. $\forall x_{0} \in W_{0}$, there exists a unique solution to the system (2.1), denoted by

$$
x\left(t ; t_{0}, x_{0}\right)= \begin{cases}x^{1}\left(t ; t_{0}, x_{0}\right), & t \in D_{1}, \\ x^{2}\left(t ; t_{f_{1}}, x^{1}\left(t_{f_{1}}\right)\right), & t \in D_{2}, \\ x^{3}\left(t ; t_{f_{2}}, x^{2}\left(t_{f_{2}}\right)\right), & t \in D_{3} .\end{cases}
$$

The solution set $S_{W_{0}}$ of the system (2.1) for different initial state vector $x_{0} \in W_{0}$, is defined as follows.

$$
\begin{aligned}
S_{W_{0}}:=\{ & x\left(\cdot ; t_{0}, x_{0}\right) \mid x\left(\cdot ; t_{0}, x_{0}\right) \text { is the solution of the system }(2.1) \\
& \text { for } \left.x_{0} \in W_{0}\right\} .
\end{aligned}
$$

It follows from (2.2)-(2.4) that there exists a definition of partial derivative of function $h^{i}\left(x^{i}(t)\right)$ with respect to $x^{i}(t) \in W_{a}$ and $h^{i}\left(x^{i}(t)\right)$ is continuous in $x^{i}(t) \in W_{a} \subset \mathbb{R}^{5}$. By Theorem 3.3 in [10], we can get an interesting property as follows.

Property 3. $\forall x\left(t_{0}\right)=x_{0} \in W_{0}$, the solution $x\left(t ; t_{0}, x_{0}\right)$ of the system $(2.1)$ is continuous in $\left(t ; t_{0}, x_{0}\right) \in D_{0} \times D_{1} \times W_{0}$, where $x^{1}\left(t ; t_{0}, x_{0}\right)$ is continuously differentiable in $\left(t ; t_{0}, x_{0}\right) \in D_{1} \times D_{1} \times W_{0} ; x^{i}\left(t ; t_{f_{i-1}}, x^{i-1}\left(t_{f_{i-1}}\right)\right)$ is continuously differentiable in $\left(t ; t_{f_{i-1}}, x^{i-1}\left(t_{f_{i-1}}\right)\right) \in D_{i} \times D_{i} \times W_{a}$, that is, $x^{1}\left(t ; t_{0}, x_{0}\right) \in$ $C^{1}\left(D_{1} \times D_{1} \times W_{0}, \mathbb{R}_{+}^{5}\right), x^{i}\left(t ; t_{f_{i-1}}, x^{i-1}\left(t_{f_{i-1}}\right)\right) \in C^{1}\left(D_{i} \times D_{i} \times W_{a}, \mathbb{R}_{+}^{5}\right), i=2,3$.

Now that every component of the solution $x\left(t ; t_{0}, x_{0}\right)$ for the system $(2.1)$ denotes the concentrations of biomass, extracellular glycerol, extracellular 1,3$\mathrm{PD}$, acetate, ethanol at time $t \in D_{0}, x\left(t ; t_{0}, x_{0}\right)$ should be restricted in a certain range according to the practical production, namely, $x\left(t ; t_{0}, x_{0}\right) \in$ $W_{\alpha} \subset \mathbb{R}_{+}^{5}, \forall t \in D_{0}$. Let $S_{W_{0 a}}$ be the solution set of satisfying the solution $x\left(t ; t_{0}, x_{0}\right) \in W_{\alpha}$,

$$
S_{W_{0 a}}:=\left\{x\left(t ; t_{0}, x_{0}\right) \in S_{W_{0}} \subset C\left(D_{0} \times D_{1} \times W_{0}, \mathbb{R}_{+}^{5}\right) \mid x\left(t ; t_{0}, x_{0}\right) \in W_{a}\right\} .
$$

It is well known that the set $S_{W_{0 a}}$ is nonempty based on numerical calculation in [24]. Next, we will prove the compactness of the solution sets $S_{W_{0}}$ and $S_{W_{0 a}}$ for the system $(2.1)$.

Theorem 1. The sets $S_{W_{0}}$ and $S_{W_{0 a}}$, defined by (2.7) and (2.8), are all compact in $C^{1}\left(D_{0} \times D_{1} \times W_{0}, \mathbb{R}_{+}^{5}\right)$ and are all convex in initial state vector $x_{0} \in W_{0}$. 
Proof. It follows from (2.5) that the set $W_{0} \subset \mathbb{R}_{+}^{5}$ is nonempty and compact. By Property 3 , we have that the mapping $x_{0} \in W_{0} \mapsto x\left(t, t_{0}, x_{0}\right) \in S_{W_{0}}$, defined by (2.6), is continuous. Thus, $S_{W_{0}}$ is nonempty and compact in $C^{1}\left(D_{0} \times D_{1} \times\right.$ $\left.W_{0}, \mathbb{R}_{+}^{5}\right) . \quad \forall x_{0}, y_{0} \in W_{0}$ and $\forall \tau \in(0,1)$, by the convexity of $W_{0}$, we have $x_{0}+\tau\left(y_{0}-x_{0}\right) \in W_{0}$. By Property 2 , the solution $x\left(t ; t_{0}, x_{0}+\tau\left(y_{0}-x_{0}\right)\right) \in S_{W_{0}}$ of the system (2.1) is existent, where $x\left(t_{0}\right)=x_{0}+\tau\left(y_{0}-x_{0}\right)$ is the initial state vector of the system (2.1), so $S_{W_{0}}$ is convex in $x_{0} \in W_{0}$.

It follows from (2.8) that $S_{W_{0 a}} \subset S_{W_{0}}$. Let $\left\{x^{k}\left(t ; t_{0}, x_{0}^{k}\right)\right\}_{k=1}^{\infty}$ be any sequence of $S_{W_{0 a}}$, namely, $\left\{x^{k}\left(t ; t_{0}, x_{0}^{k}\right)\right\}_{k=1}^{\infty}$ is the sequence of compact set $S_{W_{0}}$. Thus, there exists a convergent subsequence in compact set $S_{W_{0}}$, denoted by $\left\{x^{k_{j}}\left(t ; t_{0}, x_{0}^{k_{j}}\right)\right\}_{k_{j}=1}^{\infty}$. The subsequence satisfies: $x^{k_{j}}\left(t ; t_{0}, x_{0}^{k_{j}}\right) \rightarrow \bar{x}\left(t ; t_{0}, \bar{x}_{0}\right)$, $x_{0}^{k_{j}} \rightarrow \bar{x}_{0}$, as $k_{j} \rightarrow \infty . W_{0} \subset \mathbb{R}^{5}$ is a closed set and $x_{0}^{k_{j}} \in W_{0}, \forall k_{j}$, so $\bar{x}_{0} \in W_{0}$.

Because $x^{k_{j}}(t)=x^{k_{j}}\left(t ; t_{0}, x_{0}^{k_{j}}\right) \in S_{W_{0 a}}$, is the solution of the system (2.1), it satisfies state equation and initial conditions as follows:

$$
\left\{\begin{array}{l}
\dot{x}^{k_{j}, i}(t)=h^{i}\left(x^{k_{j}, i}\right), t \in D_{i}, i \in I_{3}, \\
x^{k_{j}, i}\left(t_{f_{i-1}}\right)=\left\{\begin{array}{l}
x^{k_{j}, 0}\left(t_{0}\right)=x_{0}^{k_{j}}, i=1, \\
x^{k_{j}, i-1}\left(t_{f_{i-1}}\right), i=2,3,
\end{array}\right. \\
x^{k_{j}}\left(t ; t_{0}, x_{0}^{k_{j}}\right) \in W_{a}, \text { namely, } x_{* l} \leq x_{l}^{k_{j}}\left(t ; t_{0}, x_{0}^{k_{j}}\right) \leq x_{l}^{*}, l \in I_{5} .
\end{array}\right.
$$

By Properties 1-3, $x^{k_{j}}\left(t ; t_{0}, x_{0}^{k_{j}}\right)$ is continuous in $x_{0}^{k_{j}} \in W_{0}, h^{i}\left(x^{k_{j}, i}\left(t ; t_{0}, x_{0}^{k_{j}}\right)\right)$ is continuous in $x^{k_{j}, i}\left(t ; t_{0}, x_{0}^{k_{j}}\right) \in W_{\alpha}$. As $k_{j} \rightarrow \infty$, it follows from (2.9) that

$$
\begin{cases}\dot{\bar{x}}^{i}\left(t ; t_{0}, \bar{x}_{0}\right)=h^{i}\left(\bar{x}\left(t ; t_{0}, \bar{x}_{0}\right)\right), & t \in D_{i}, i \in I_{3}, \\ \bar{x}^{i}\left(t_{f_{i-1}}\right)= \begin{cases}\bar{x}\left(t_{0}\right)=\bar{x}_{0}, & i=1, \\ \bar{x}^{i-1}\left(t_{f_{i-1}}\right), & i=2,3\end{cases} \end{cases}
$$

and $x_{* l} \leq \bar{x}_{l}\left(t ; t_{0}, \bar{x}_{0}\right) \leq x_{l}^{*}, l \in I_{5}$. It follows from (2.8) that $\bar{x}\left(t ; t_{0}, \bar{x}_{0}\right) \in S_{W_{0 a}}$. This means that subsequence $\left\{x^{k_{j}}\left(t ; t_{0}, x_{0}^{k_{j}}\right)\right\}_{k_{j}=1}^{\infty}$ is convergent in $S_{W_{0 a}}$ and its limitation satisfies $\bar{x}\left(t ; t_{0}, \bar{x}_{0}\right) \in S_{W_{0 a}}$. Thus, the function set $S_{W_{0 a}}$ is compact in $C^{1}\left(D_{0} \times D_{1} \times W_{0}, \mathbb{R}_{+}^{5}\right)$. By the definition of convex set, we can prove that $S_{W_{0 a}}$ is convex in $x_{0} \in W_{0}$.

\section{Linear variational system and its fundamental matrix solution}

Now that partial derivative of function $h^{i}\left(x^{i}\right)$ is existent for $x^{i} \in W_{a}$ and continuous in $t \in D_{0}$, it follows that we can structure corresponding linear variational systems (3.1)-(3.3) for the solution to the system (2.1).

$$
\begin{aligned}
\dot{y}^{1}(t) & =\frac{\partial h^{1}\left(x^{1}\left(t ; t_{0}, x_{0}\right)\right)}{\partial x^{1}} y^{1}(t), \quad t \in D_{1}, \\
\dot{y}^{2}(t) & =\frac{\partial h^{2}\left(x^{2}\left(t ; t_{f_{1}}, x^{1}\left(t_{f_{1}} ; t_{0}, x_{0}\right)\right)\right)}{\partial x^{2}} y^{2}(t), \quad t \in D_{2}, \\
\dot{y}^{3}(t) & =\frac{\partial h^{3}\left(x^{3}\left(t ; t_{f_{2}}, x^{2}\left(t_{f_{2}} ; t_{0}, x^{1}\left(t_{f_{1}} ; t_{0}, x_{0}\right)\right)\right)\right)}{\partial x^{3}} y^{3}(t), t \in D_{3},
\end{aligned}
$$


where $x^{1}\left(t ; t_{0}, x_{0}\right) \in \mathbb{R}^{5}$ is the solution of the first stage for system (2.1); $x^{i}\left(t ; t_{f_{i-1}}, x^{i-1}\left(t_{f_{i-1}}\right)\right), i=2,3$ is the solution of the $i_{t h}=2,3$ stages for the system (2.1).

By Theorem 3.3 in $[10], x^{1}(t)=x^{1}\left(t ; t_{0}, x_{0}\right)$ is the solution to the state equation (3.4) of the first stage for the system (2.1)

$$
\left\{\begin{array}{l}
\dot{x}^{1}(t)=h^{1}\left(x^{1}(t)\right), t \in D_{1} \\
x^{1}\left(t_{0}\right)=x_{0}
\end{array}\right.
$$

so matrix $\frac{\partial x^{1}\left(t ; t_{0}, x_{0}\right)}{\partial x_{0}} \in \mathbb{R}^{5 \times 5}$ is the fundamental matrix solution of linear variable system (3.1) with initial state vector

$$
\frac{\partial x^{1}\left(t_{0} ; t_{0}, x_{0}\right)}{\partial x_{0}}=I, \quad\left(I \in \mathbb{R}^{5 \times 5} \text { unit matrix }\right) .
$$

Similarly, for $i=2,3, x^{i}(t)=x^{i}\left(t ; t_{f_{i-1}}, x^{i-1}\left(t_{f_{i-1}}\right)\right)$ is the solution of the state equation (3.6) of the $i_{t h}$ stage for the system (2.1)

$$
\begin{cases}\dot{x}^{i}(t) & =h^{i}\left(x^{i}(t)\right), t \in D_{i} \\ x^{i}\left(t_{f_{i-1}}\right) & =x^{i-1}\left(t_{f_{i-1}}\right) .\end{cases}
$$

Therefore, matrix $\frac{\partial x^{i}\left(t ; t_{f_{i-1}}, x^{i-1}\left(t_{f_{i-1}}\right)\right)}{\partial x^{i-1}\left(t_{f_{i-1}}\right)} \in \mathbb{R}^{5 \times 5}$ is the fundamental matrix solution of linear variational system (3.2) and (3.3) with initial state vector

$$
\frac{\partial x^{i}\left(t_{f_{i-1}} ; t_{f_{i-1}}, x^{i-1}\left(t_{f_{i-1}}\right)\right)}{\partial x^{i-1}\left(t_{f_{i-1}}\right)}=I, \quad\left(I \in \mathbb{R}^{5 \times 5} \text { unit matrix }\right), i=2,3 .
$$

Let $\Phi^{1}\left(t ; t_{0}, x_{0}\right), \Phi^{2}\left(t ; t_{f_{1}}, x^{1}\left(t_{f_{1}}\right)\right)$ and $\Phi^{3}\left(t ; t_{f_{2}}, x^{2}\left(t_{f_{2}}\right)\right)$ are the fundamental matrix solutions of the linear variable systems (3.1)-(3.3) with initial state (3.5)(3.7), respectively. By Theorem 2.6.4 in [12], we give the following lemma

Lemma 1. Let $x^{1}\left(t ; t_{0}, x_{0}\right)$ and $y^{1}\left(t ; t_{0}, y_{0}\right)$ be the solutions to state equation (3.4) of the first stage for the system (2.1) with the given initial state vectors $x^{1}\left(t_{0} ; t_{0}, x_{0}\right)=x_{0} \in W_{0}$ and $y^{1}\left(t_{0} ; t_{0}, y_{0}\right)=y_{0} \in W_{0}$, respectively. Then,

$$
y^{1}\left(t ; t_{0}, y_{0}\right)-x^{1}\left(t ; t_{0}, x_{0}\right)=\int_{0}^{1} \Phi^{1}\left(t ; t_{0}, x_{0}+\tau\left(y_{0}-x_{0}\right)\right) d \tau \cdot\left(y_{0}-x_{0}\right), \forall t \in D_{1} .
$$

Similarly, let $x^{i}\left(t ; t_{f_{i-1}}, x^{i-1}\left(t_{f_{i-1}}\right)\right)$ and $y^{i}\left(t ; t_{f_{i-1}}, y^{i-1}\left(t_{f_{i-1}}\right)\right)$ be the solutions to state equation (3.6) of the $i_{t h}=2,3$ stage for the system (2.1) with initial state vector $x^{i}\left(t_{f_{i-1}}\right)=x^{i-1}\left(t_{f_{i-1}}\right) \in W_{0}$ and $y^{i}\left(t_{f_{i-1}}\right)=y^{i-1}\left(t_{f_{i-1}}\right) \in W_{0}$, respectively. Then, $\forall t \in D_{i}, i=2,3$,

$$
\begin{aligned}
& y^{i}\left(t ; t_{f_{i-1}}, y^{i-1}\left(t_{f_{i-1}}\right)\right)-x^{i}\left(t ; t_{f_{i-1}}, x^{i-1}\left(t_{f_{i-1}}\right)\right) \\
& =\int_{0}^{1} \Phi^{i}\left(t ; t_{f_{i-1}}, x^{i-1}\left(t_{f_{i-1}}\right)+\tau\left(y^{i-1}\left(t_{f_{i-1}}\right)-x^{i-1}\left(t_{f_{i-1}}\right)\right)\right) d \tau \\
& \cdot\left(y^{i-1}\left(t_{f_{i-1}}\right)-x^{i-1}\left(t_{f_{i-1}}\right)\right) .
\end{aligned}
$$




\section{Strong stability of nonlinear multi-stage dynamic system}

The purpose of the section is to discuss the strong stability with respect to initial state vector for the solution to the system (2.1). Now, we introduce its definition.

\section{Definition 1. Let}

$$
x(t)=x\left(t ; t_{0}, x_{0}\right):=\left[x^{1}\left(t ; t_{0}, x_{0}\right), x^{2}\left(t ; t_{f_{1}}, x^{1}\left(t_{f_{1}}\right)\right), x^{3}\left(t ; t_{f_{2}}, x^{2}\left(t_{f_{2}}\right)\right)\right]^{\mathrm{T}}
$$

be the solution of system (2.1) with initial state vector $x^{1}\left(t_{0} ; t_{0}, x_{0}\right)=x_{0} \in$ $W_{0}$. For $\forall \epsilon>0$, if there exists a $\delta=\delta(\epsilon)>0$ such that the solution $y(t)=y\left(t ; t_{0}, y_{0}\right):=\left[y^{1}\left(t ; t_{0}, y_{0}\right), y^{2}\left(t ; t_{f_{1}}, y^{1}\left(t_{f_{1}}\right)\right), y^{3}\left(t ; t_{f_{2}}, y^{2}\left(t_{f_{2}}\right)\right)\right]^{\mathrm{T}}$ of the system (2.1) with initial state vector $y^{1}\left(t_{0} ; t_{0}, y_{0}\right)=y_{0} \in W_{0}$ satisfies: $y_{0} \in$ $W_{0},\left|x_{0}-y_{0}\right|<\delta,|x(t)-y(t)|<\epsilon, \forall t \in D_{0}$. That is,

$$
\begin{aligned}
& \left|x^{1}\left(t ; t_{0}, x_{0}\right)-y^{1}\left(t ; t_{0}, y_{0}\right)\right|<\epsilon, \forall t \in D_{1}, \\
& \left|x^{i}\left(t ; t_{f_{i-1}}, x^{i-1}\left(t_{f_{i-1}}\right)\right)-y^{i}\left(t ; t_{f_{i-1}}, y^{i-1}\left(t_{f_{i-1}}\right)\right)\right|<\epsilon, \forall t \in D_{i}, \quad i=2,3 .
\end{aligned}
$$

Then, the solution $x\left(t ; t_{0}, x_{0}\right)$ of the system (2.1) is said to be strong stable with respect to initial state vector $x_{0} \in W_{0}$.

To prove the strong stability of the solution for the system (2.1), we need prove the boundedness of the fundamental matrix solution for the linear variational systems (3.1)-(3.3).

Theorem 2. Let $x^{1}\left(t ; t_{0}, x_{0}\right)$ be the solution of state equation (3.4) of the first stage for the system (2.1) with initial state $x^{1}\left(t_{0} ; t_{0}, x_{0}\right)=x_{0} \in W_{0}$ and $\Phi^{1}\left(t ; t_{0}, x_{0}\right), t \in D_{1}$ the fundamental matrix solution for the linear variational systems (3.1), then $\Phi^{1}\left(t ; t_{0}, x_{0}\right)$ is bounded in $D_{1} \subset \mathbb{R}_{+}$. That is, there exists a constant $M>0$, such that

$$
\left|\Phi^{1}\left(t ; t_{0}, x_{0}\right)\right| \leq M, \forall t \in D_{1} .
$$

Proof. Let $\Phi^{1}\left(t ; t_{0}, x_{0}\right):=\left[y^{1}\left(t ; t_{0}, e^{1}\right), y^{2}\left(t ; t_{0}, e^{2}\right), \ldots, y^{5}\left(t ; t_{0}, e^{5}\right)\right] \in \mathbb{R}^{5 \times 5}$. By the definition of $\Phi^{1}\left(t ; t_{0}, x_{0}\right)$, we get

$$
\left\{\begin{array}{l}
\dot{y}^{j}\left(t ; t_{0}, e^{j}\right)=\frac{\partial h^{1}\left(x^{1}\left(t ; t_{0}, x_{0}\right)\right)}{\partial x^{1}} y^{j}\left(t ; t_{0}, e^{j}\right), t \in D_{1}, j \in I_{5}, \\
y^{j}\left(t_{0} ; t_{0}, e^{j}\right)=e^{j}, j \in I_{5}
\end{array}\right.
$$

where $e^{j} \in \mathbb{R}^{5}$ is an unit vector which the $j_{t h} \in I_{5}$ component is 1 , other components are 0 . Let

$$
\mathbf{y}^{j}(t):=\left[\mathbf{y}_{1}^{j}(t), \mathbf{y}_{2}^{j}(t), \ldots, \mathbf{y}_{5}^{j}(t)\right]^{\mathrm{T}}, j \in I_{5}
$$

be the solution of the system (4.1).

Since partial derivative of function $h^{i}\left(x^{i}\right)$ is existent for $x^{i} \in W_{a}$ and continuous in $t \in D_{0}$ as well as $W_{a} \subset \mathbb{R}^{5}$ is a nonempty and compact set, 
$\frac{\partial h^{1}\left(x\left(t ; t_{0}, x_{0}\right)\right)}{\partial x^{1}}$ is bounded in $t \in D_{1}$ for given $\left(t_{0}, x_{0}\right) \in D_{1} \times W_{0}$. That is, $\exists M_{1}>0$, such that

$$
\left|\frac{\partial h^{1}\left(x^{1}\left(t ; t_{0}, x_{0}\right)\right)}{\partial x^{1}}\right| \leq M_{1}, \quad \forall t \in D_{1} .
$$

Let

$$
\begin{aligned}
& u_{j}(t):=\arg \min \left\{\left|u_{j}(t)\right|: u_{j}(t) \in C^{1}\left(D_{1}, \mathbb{R}_{+}\right), u_{j}(t) \geq \max _{1 \leq l \leq 5}\left|\mathbf{y}_{l}^{j}(t)\right|\right\}, \\
& W_{j}\left(t ; u_{j}(t)\right):=5 M_{1} u_{j}(t), \quad j \in I_{5} .
\end{aligned}
$$

Clearly, $W_{j}\left(t ; u_{j}(t)\right)$ is continuous in $D_{1} \times \mathbb{R}_{+}$, so there exists an unique solution $u_{j}(t) \geq 1, t \in D_{1}$ to system (4.4)

$$
\left\{\begin{array}{l}
\dot{u}_{j}(t)=W_{j}\left(t ; u_{j}(t)\right), t \in D_{1}, \\
u_{j}\left(t_{0}\right)=1, j \in I_{5}
\end{array}\right.
$$

For the right item of state equation for the system (4.1), due to

$$
\left|y^{j}\left(t_{0} ; t_{0}, e^{j}\right)\right|=\left|e^{j}\right|=1, \quad u_{j}\left(t_{0}\right)=1, \quad j \in I_{5},
$$

we have

$$
\begin{aligned}
\left|\frac{\partial h^{1}\left(x^{1}\left(t ; t_{0}, x_{0}\right)\right)}{\partial x^{1}} \mathbf{y}^{j}(t)\right| & \leq\left|\frac{\partial h^{1}\left(x\left(t ; t_{0}, x_{0}\right)\right)}{\partial x^{1}}\right|\left|\mathbf{y}^{j}(t)\right|(\text { Based on (4.2), we have) } \\
& \leq M_{1}\left|\mathbf{y}^{j}(t)\right|(\text { Based on the definition of norm, we have) } \\
& \leq 5 M_{1} \max _{1 \leq l \leq 5}\left|\mathbf{y}_{l}^{j}(t)\right|(\text { Based on (4.3), we have) } \\
& \leq 5 M_{1} u_{j}(t)=W_{j}\left(t ; u_{j}(t)\right), \quad \forall t \in D_{1}, j \in I_{5}
\end{aligned}
$$

which implies

$$
\left|\frac{\partial h^{1}\left(x^{1}\left(t ; t_{0}, x_{0}\right)\right)}{\partial x^{1}} \mathbf{y}^{j}(t)\right| \leq W_{j}\left(t, u_{j}(t)\right), \quad j \in I_{5} .
$$

Comparing the systems (4.1) with (4.4), by Theorem 6.1 and Corollary 6.3 in [12], we get that the solution of the system (4.1) satisfies

$$
\left|y^{j}\left(t ; t_{0}, e^{j}\right)\right| \leq u_{j}(t) \leq\left|u_{j}(t)\right|=\max _{t \in D_{1}} u_{j}(t), \quad j \in I_{5} .
$$

By $u_{j}(t) \in C^{1}\left(D_{1}, \mathbb{R}_{+}\right), u_{j}(t)$ is bounded in $D_{1} \subset \mathbb{R}_{+}$, namely, $\left|u_{j}(t)\right| \leq m_{j}<\infty$. By (4.5), we obtain that the solution $y^{j}\left(t ; t_{0}, e^{i}\right)$ of system (4.1) satisfies

$$
\begin{gathered}
\left|y^{j}\left(t ; t_{0}, e^{j}\right)\right| \leq m_{j}, \quad \forall t \in D_{1}, j \in I_{5} . \\
\left|\Phi^{1}\left(t ; t_{0}, x_{0}\right)\right|=\left|\left[y^{1}\left(t ; t_{0}, e^{1}\right), y^{2}\left(t ; t_{0}, e^{2}\right), \ldots, y^{5}\left(t ; t_{0}, e^{5}\right)\right]\right| \\
=\sum_{j=1}^{5} \sum_{l=1}^{5}\left|y_{l}^{j}\left(t ; t_{0}, e^{j}\right)\right| \leq \sum_{j=1}^{5} m_{j}, \quad \forall t \in D_{1} .
\end{gathered}
$$


Let $M=\sum_{j=1}^{5} m_{j}$, then

$$
\left|\Phi^{1}\left(t ; t_{0}, x_{0}\right)\right| \leq M, t \in D_{1},
$$

which completes our proof.

Using the same argument as in the proof of Theorem 2, we can easily carry out the proof of Theorem 3 .

Theorem 3. The fundamental matrix solutions $\Phi^{2}\left(t ; t_{f_{1}}, x^{1}\left(t_{f_{1}}\right)\right)$ and $\Phi^{3}\left(t ; t_{f_{2}}, x^{1}\left(t_{f_{2}}\right)\right)$ of the linear variational systems (3.2) and (3.3) are bounded in $D_{2}$ and $D_{3}$, respectively. Thus, there exists a constant $M>0$, such that

$$
\left|\Phi^{i}\left(t ; t_{f_{i-1}}, x^{i-1}\left(t_{f_{i-1}}\right)\right)\right| \leq M, \quad \forall t \in D_{i}, i=2,3 .
$$

With the help of the preceding two theorems we can prove the strong stability of the solution for the system (2.1).

Theorem 4. Let $x\left(t ; t_{0}, x_{0}\right)$ is the solution of the system (2.1) with $x\left(t_{0}\right)=$ $x_{0} \in W_{0}$. Then, the solution $x\left(t ; t_{0}, x_{0}\right)$, denoted by (2.6), is strongly stable with respect to initial state vector $x_{0} \in W_{0}$.

Proof. $\forall \epsilon>0$, let $y\left(t ; t_{0}, y_{0}\right) \in S_{W_{0 a}}$ be the solution of the system (2.1) with

$$
y_{0} \in W_{0}, \quad\left|x_{0}-y_{0}\right|<\frac{\epsilon}{M}=\delta(\epsilon), \quad\left|y^{i}\left(t_{f_{i}}\right)-x^{i}\left(t_{f_{i}}\right)\right|<\delta(\epsilon),
$$

where $M>0$ is a constant in Theorems 2 and 3. By Lemma 1, we have

$$
=\left\{\begin{array}{c}
y\left(t ; t_{0}, y_{0}\right)-x\left(t ; t_{0}, x_{0}\right)= \\
\int_{0}^{1} \Phi^{1}\left(t ; t_{0}, x_{0}+\tau\left(y_{0}-x_{0}\right)\right) \mathrm{d} \tau \cdot\left(y_{0}-x_{0}\right), \forall t \in D_{1}, \\
\int_{0}^{1} \Phi^{i}\left(t ; t_{f_{i-1}}, x^{i-1}\left(t_{f_{i-1}}\right)+\tau\left(y^{i-1}\left(t_{f_{i-1}}\right)-x^{i-1}\left(t_{f_{i-1}}\right)\right)\right) \mathrm{d} \tau \\
\times\left(y^{i-1}\left(t_{f_{i-1}}\right)-x^{i-1}\left(t_{f_{i-1}}\right)\right), \forall t \in D_{i}, i=2,3 .
\end{array}\right.
$$

Since $W_{0} \subset \mathbb{R}^{5}$ is a convex and compact set, it follows that if $x_{0} \in W_{0}, y_{0} \in W_{0}$, then, $\forall \tau \in(0,1), x_{0}+\tau\left(y_{0}-x_{0}\right) \in W_{0}$. Taking norm to (4.7) gives

$$
\begin{gathered}
\quad\left|y\left(t ; t_{0}, y_{0}\right)-x\left(t ; t_{0}, x_{0}\right)\right| \leq \\
\left|\int_{0}^{1} \Phi^{1}\left(t ; t_{0}, x_{0}+\tau\left(y_{0}-x_{0}\right)\right) \mathrm{d} \tau\right|\left|\left(y_{0}-x_{0}\right)\right|, \quad \forall t \in D_{1}, \\
\left|\int_{0}^{1} \Phi^{i}\left(t ; t_{f_{i-1}}, x^{i-1}\left(t_{f_{i-1}}\right)+\tau\left(y^{i-1}\left(t_{f_{i-1}}\right)-x^{i-1}\left(t_{f_{i-1}}\right)\right)\right) \mathrm{d} \tau\right| \\
\quad \times\left|\left(y^{i-1}\left(t_{f_{i-1}}\right)-x^{i-1}\left(t_{f_{i-1}}\right)\right)\right|, \quad \forall t \in D_{i}, i=2,3 .
\end{gathered}
$$


By Theorems 2 and 3 together with (4.6), we have

$$
\left|y\left(t ; t_{0}, y_{0}\right)-x\left(t ; t_{0}, x_{0}\right)\right| \leq\left\{\begin{array}{l}
M\left|\left(y_{0}-x_{0}\right)\right| \leq \epsilon, \quad \forall t \in D_{1} \\
M\left|\left(y^{i-1}\left(t_{f_{i-1}}\right)-x^{i-1}\left(t_{f_{i-1}}\right)\right)\right| \leq \epsilon \\
\forall t \in D_{i}, \quad i=2,3
\end{array}\right.
$$

namely, $\left|y\left(t ; t_{0}, y_{0}\right)-x\left(t ; t_{0}, x_{0}\right)\right| \leq \epsilon, \forall t \in D_{0}$. By Definition 1 , this completes the proof of Theorem 4 .

\section{Numerical simulation}

A batch culture was carried out under anaerobic conditions at $37^{\circ} \mathrm{C}$ with 4 groups initial state vectors, i.e.,

$x_{0}^{1}=[0.102,418.2609,0,0,0], x_{0}^{2}=[0.2025,441.337,0,0,0]$,

$x_{0}^{3}=[0.173,402.9348,0,0,0], x_{0}^{4}=[0.2245,509.8913,0,0,0], \Delta x_{0}^{l}=0.1 x_{0}^{l}$,

$l \in I_{4}$. The system (2.1) was numerical solved by using the Euler method. The step size of the Euler method was 1/72000 h, which was derived empirically after several numerical experiments.
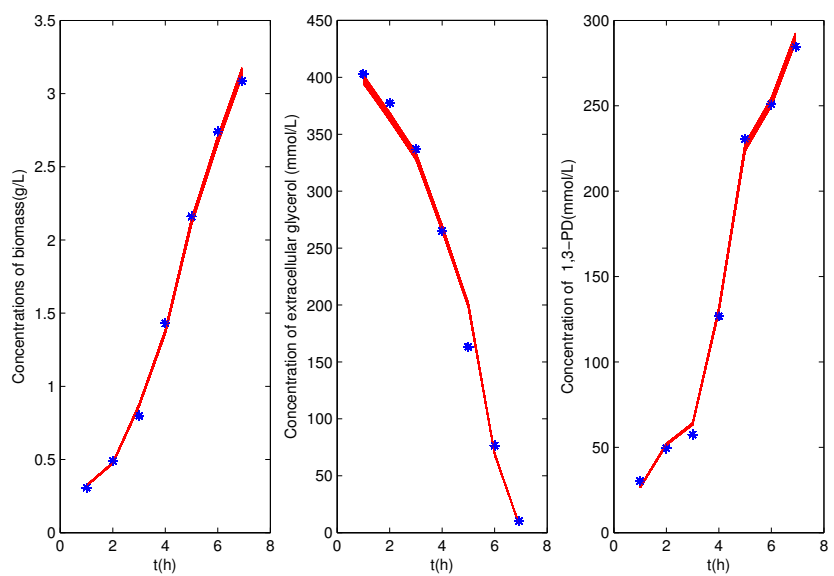

Figure 1. Comparison of biomass, glycerol, 1,3-PD concentration between experimental data and computational results $x\left(t, x_{0}^{1}+\rho^{i} \Delta x_{0}^{1}\right),\left|\rho^{i}\right|<1, i \in I_{N}, N=1000$, with $x_{0}^{1}=[0.102,418.2609,0,0,0]$.

Figures 1-4 show the comparison of the first three components between experimental data and computational results $x\left(t, x_{0}^{l}+\rho^{i} \Delta x_{0}^{l}\right),\left|\rho^{i}\right|<1, l \in$ $I_{4}, i \in I_{N},\left(N\right.$ is the times of perturbation with initial state vector $\left.x_{0}^{l}\right)$ where the stars denote the experimental data and the solid lines denote the computational curves. From Figures 1-4, we can deduce the strong stability of the system (2.1). 

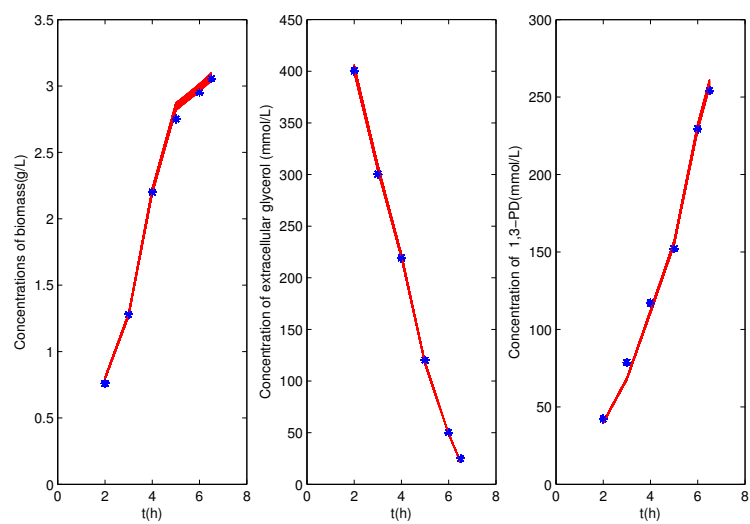

Figure 2. Comparison of biomass, glycerol, 1,3-PD concentration between experimental data and computational results $x\left(t, x_{0}^{2}+\rho^{i} \Delta x_{0}^{2}\right),\left|\rho^{i}\right|<1, i \in I_{N}, N=1000$, with $x_{0}^{2}=[0.2025,441.337,0,0,0]$.
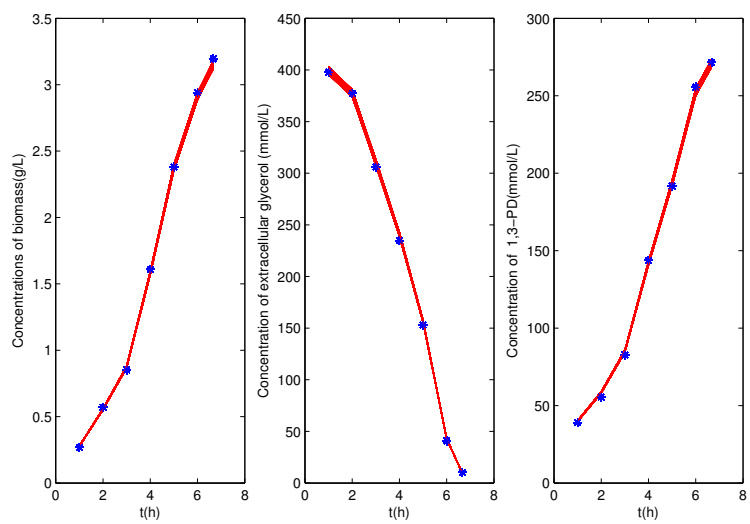

Figure 3. Comparison of biomass, glycerol, 1,3-PD concentration between experimental data and computational results $x\left(t, x_{0}^{3}+\rho^{i} \Delta x_{0}^{3}\right),\left|\rho^{i}\right|<1, i \in I_{N}, N=1000$, with $x_{0}^{3}=[0.173,402.9348,0,0,0]$.

\section{Conclusions}

This paper has studied the strong stability with respect to initial state for the solution to the nonlinear multi-stage dynamic system in which there is no equilibrium point. In addition, we structure corresponding linear variational system for the solution to the multi-stage system. Then, a sufficient condition on this strong stability are given by the boundedness to the fundamental matrix solution of this linear variational system. Finally, numerical simulations are 

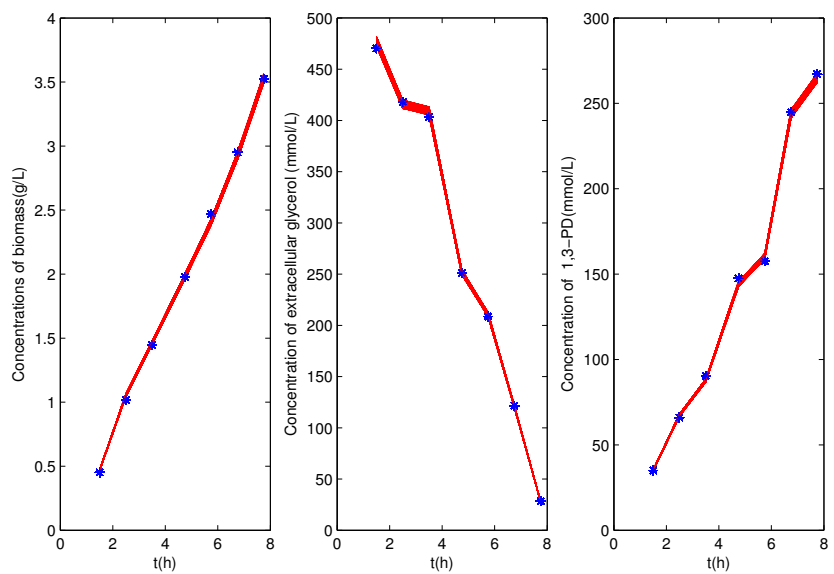

Figure 4. Comparison of biomass, glycerol, 1,3-PD concentration between experimental data and computational results $x\left(t, x_{0}^{4}+\rho^{i} \Delta x_{0}^{4}\right),\left|\rho^{i}\right|<1, i \in I_{N}, N=1000$, with $x_{0}^{4}=[0.2245,509.8913,0,0,0]$.

agreement with theoretical analysis, both of which confirm the correctness of the presented theory.

The current work deals with the problem of strong stability of the multistage system in batch culture. In a future work, our effort will focus on optimal control of the multi-stage system.

\section{Acknowledgements}

This work was supported by the National Natural Science Foundation of China (Grant Nos. 11171050 and 11371164), the National Science Foundation for the Youth of China (Grant Nos. 11301051, 11301081 and 11501574), the Provincial Natural Science Foundation of Fujian (Grant No. 2014J05001), the Fundamental Research Funds for Central Universities in China (Grant No. DUT15LK25), Natural Science Foundation of Shandong Province in China (Grant No. ZR2015AL010) and the China Scholorship Council (CSC, No. 201506060121).

\section{References}

[1] A. Ashoori, B. Moshiri, A.Khaki-Sedigh and M.R. Bakhtiari. Optimal control of a nonlinear fed-batch fermentation process using model predictive approach. Journal Process Control, 19(7):1162-1173, 2009. http://dx.doi.org/10.1016/j.jprocont.2009.03.006.

[2] G. Cheng, L. Wang, R. Loxton and Q. Lin. Robust optimal control of a microbial batch culture process. Journal of Optimization Theory and Applications, 167(1):342-362, 2015. http://dx.doi.org/10.1007/s10957-014-0654-z. 
[3] S.-K. Choi, Y.-H. Cui and N. Koo. Variationally stable dynamic systems on time scales. Advances of Difference Equations, 2012(1):129, 2012. http://dx.doi.org/10.1186/1687-1847-2012-129.

[4] S.-K. Choi and N. Koo. On the stablility of linear dynamic system on time scales. Journal of Difference Equations Applications, 15(2):167-183, 2009. http://dx.doi.org/10.1080/10236190802008528.

[5] E. Fridman. Stability of linear descriptor systems with delay: a Lyapunov-based approach. Journal of Mathematical Analysis and Applications, 273(1):24-44, 2002. http://dx.doi.org/10.1016/S0022-247X(02)00202-0.

[6] K. Gao, X. Zhang, E. Feng and Z. Xiu. Sensitivity analysis and parameter identification of nonlinear hybrid systems for glycerol transport mechanisms in continuous culture. Journal of Theoretical Biology, 347:137-143, 2014. http://dx.doi.org/10.1016/j.jtbi.2013.12.025.

[7] Y. Gao, J. Lygeros and M. Quincampoix. On the reachability problem for uncertain hybrid systems. IEEE Transactions on Automatic Control, 52(9):1572-1586, 2007. http://dx.doi.org/10.1109/TAC.2007.904449.

[8] Y. Gao, J. Lygeros, M. Quincampoix and N. Seube. Approximate stabilisation of uncertain hybrid systems. Hybrid Systems: Computation and Control, 2623:203215, 2003. http://dx.doi.org/10.1007/3-540-36580-X_17.

[9] S. Gottlieb, C.W. Shu and E. Tadmor. Strong stability-preserving highorder time discretization methods. SIAM review, 43(1):89-112, 2001. http://dx.doi.org/10.1137/S003614450036757X.

[10] D.P. Hou. Ordinary differential equation. People's Education Press, 1980.

[11] D. Klatte and K. Tammer. Strong stability of stationary solutions and KarushKuhn-Tucker points in nonlinear optimization. Annals of Operations Research, 27(1):285-307, 1990. http://dx.doi.org/10.1007/BF02055199.

[12] V. Lakshmikantham and S. Leela. Differential and Integral Inequalities. Theory and Applications. Academic Press, New York, 1969.

[13] X. Li, E. Feng and Z. Xiu. Stability analysis of equilibrium for microorganisms in continuous culture. Applied Mathematics-A Journal of Chinese Universities, 20(4):377-383, 2005. http://dx.doi.org/10.1007/s11766-005-0014-8.

[14] X. Li, R. Qu and E. Feng. Stability and Hopf bifurcation of a delay differential system in microbial continuous culture. International Journal of Biomathematics, 02(03):321-338, 2009. http://dx.doi.org/10.1142/S179352450900073X.

[15] Z. Li, R. Zhang, S. Xu, Y. Qian and J. Xu. Stability analysis of dynamic collaboration model with control signals on two lanes. Communications in Nonlinear Science and Numerical Simulation, 19(12):4148-4160, 2014. http://dx.doi.org/10.1016/j.cnsns.2014.04.021.

[16] C. Liu. Modelling and parameter identification for a nonlinear time-delay system in microbial batch fermentation. Applied Mathematical Modelling, 37(1011):6899-6908, 2013. http://dx.doi.org/10.1016/j.apm.2013.02.021.

[17] C. Liu and Z. Gong. Modelling and optimal control of a time-delayed switched system in fed-batch process. Journal of the Franklin Institute, 351(2):840-856, 2014. http://dx.doi.org/10.1016/j.jfranklin.2013.09.014.

[18] J. Liu. Strong stability in variational inequalities. SIAM Journal on Control and Optimization, $\mathbf{3 3 ( 3 ) : 7 2 5 - 7 4 9 ,} 1995$. http://dx.doi.org/10.1137/S0363012992240527. 
[19] M. Morley. Categoricity in power. Transactions of the American Mathematical Society, 114(2):514-538, 1965. http://dx.doi.org/10.1090/S0002-9947-19650175782-0.

[20] S. Shelah. Stable theories. Israel Journal of Mathematics, 7(3):187-202, 1969. http://dx.doi.org/10.1007/BF02787611.

[21] S. Shelah. Classification theory and the number of nonisomorphic models. Studies in Logic and the Foundations of Mathematics (2nd ed.), Elsevier, 1990.

[22] R.-J. Spiteri and S.-J. Ruuth. A new class of optimal high-order strong-stabilitypreserving time discretization methods. SIAM Journal on Numerical Analysis, 40(2):469-491, 2002. http://dx.doi.org/10.1137/S0036142901389025.

[23] J. Wang, J. Ye, H. Yin, E. Feng and L. Wang. Sensitivity analysis and identification of kinetic parameters in batch fermentation of glycerol. Journal of Computational and Applied Mathematics, 236(9):2268-2276, 2012. http://dx.doi.org/10.1016/j.cam.2011.11.015.

[24] L. Wang. Determining the transport mechanism of an enzyme-catalytic complex metabolic network based on biological robustness. Bioprocess and Biosystems Engineering, 36(4):433-441, 2013. http://dx.doi.org/10.1007/s00449-012-08007.

[25] L. Wang, Q. Lin, R. Loxton, K.-L. Teo and G. Cheng. Optimal 1, 3-propanediol production: Exploring the trade-off between process yield and feeding rate variation. J. Process Control, 32:1-9, 2015. http://dx.doi.org/10.1016/j.jprocont.2015.04.011.

[26] U. Witt, R.-J. Müller, J. Augusta, H. Widdecke and W.-D. Deckwer. Synthesis, properties and biodegradability of polyesters based on 1,3-propanediol. Macromol. Chem. Phys., 195(2):793-802, 1994. http://dx.doi.org/10.1002/macp.1994.021950235.

[27] Z.-L. Xiu, A.-P. Zeng and W.-D. Deckwer. Multiplicity and stability analysis of microorganisms in continuous culture: Effects of metabolic overflow and growth inhibition. Biotechnology and Bioengineering, 57(3):251261, 1998. http://dx.doi.org/10.1002/(SICI)1097-0290(19980205)57:3ز251::AIDBIT1¿3.0.CO;2-G.

[28] J. Yuan, X. Zhang, X. Zhu, E. Feng, H. Yin, Z. Xiu and B. Tan. Identification and robustness analysis of nonlinear multi-stage enzyme-catalytic dynamical system in batch culture. Computational and Applied Mathematics, 34(3):957-978, 2015. http://dx.doi.org/10.1007/s40314-014-0160-9.

[29] A.-P. Zeng. A kinetic model for product formation of microbial and mammalian cells. Biotechnology and Bioengineering, 46(4):314-324, 1995. http://dx.doi.org/10.1002/bit.260460404.

[30] H. Zhao and E. Feng. $\phi_{0}$-stability of an impulsive system obtained from perturbing Lyapunov functions. Nonlinear Analysis: Theory, Methods $\&$ Applications, 66(4):962-967, 2007. http://dx.doi.org/10.1016/j.na.2005.12.034. 